\title{
LA DIMENSIÓN ÉTICA DE LA PRÁCTICA PROFESIONAL: evaluación de un programa formativo
}

\author{
A dimensão ética da prática profissional: \\ avaliação de um programa formativo
}

\begin{abstract}
Moisés Esteban Guitart
Profesor asociado y coordinador del "taller de ética profesional" del D epartament de Psicologia, Universitat de Girona, G irona, ES - España, e-mail: moises.esteban@ udg.edu
\end{abstract}

\section{Resumen}

El Colegio Oficial de Psicólogos establece el conocimiento y cumplimiento del código deontológico como requisito imprescindible para el ejercicio profesional de los psicólogos y psicólogas en sus distintos ámbitos de aplicación. El objetivo del trabajo que se presenta es comprobar los conocimientos de los estudiantes que están a punto de terminar la carrera de Psicología, en relación con distintas situaciones éticamente problemáticas en dos momentos temporales (antes y después de un programa de capacitación sobre ética y deontología). Se desarrolla un cuestionario que describe 5 situaciones profesionales. 51 estudiantes respondían dos preguntas asociadas a cada situación. Los resultados muestran una mejora significativa en las respuestas tras el programa educativo sobre ética aplicada. Se hace un llamamiento a desarrollar cursos en las Universidades de ética profesional con el fin de que los estudiantes dispongan de estrategias para identificar dilemas éticos y puedan llegar a posibles soluciones de acuerdo al Código D eontológico.

Palabras-clave: Código deontológico. Dilema ético. Educación superior. Programa formativo.

Rev. Diálogo Educ., Curitiba, v. 9, n. 26, p. 91-101, jan./ abr. 2009 


\section{Resumo}

A escola oficial dos psicólogos estabelece o conhecimento e o cumprimento do código deontológico como exigência essencial para o exercício profissional dos psicólogos e psicólogas em diferentes âmbitos de aplicação. O objetivo do trabalho é verificar o conhecimento dos estudantes que estão em vias de concluir o curso de Psicologia com relação a diferentes situações eticamente problemáticas, em dois momentos distintos (antes e depois de um programa de capacitação sobre ética e deontologia). A pesquisa se realiza por meio de um questionário que descreve cinco situações profissionais. Cinquenta e um estudantes responderam duas perguntas associadas a cada situação. Os resultados mostram uma melhoria significativa nas respostas após o programa educativo em éticas aplicadas. Isso indica a necessidade de desenvolver cursos de ética profissional nas universidades a fim de que os estudantes tenham as estratégias para identificar dilemas éticos e possam alcançar soluções possíveis de acordo com o código de Deontológico.

Palavras-chave: Código deontológico. Dilema ético. Instrução superior. Programa formativo.

La formación en ética aplicada es necesaria e imprescindible en los estudios de psicología ya que el conocimiento y cumplimiento del código deontológico es un requisito para el ejercicio profesional (Colegio Oficial de Psicólogos, 2004). Como constata la Federación Europea de Asociaciones Psicológicas (EFPA) "el psicólogo debe estar especialmente entrenado para abordar dilemas éticos dentro de la comunicación abierta con sus clientes, colegas y terceras personas relevantes" (CO LEGIO OFICIAL D E PSICÓ LOG O S, 2004, p. 131). No obstante, la formación en ética tiene poca presencia en los planes de estudio de la carrera de psicología. Ello impide que los futuros psicólogos y psicólogas dispongan de los recursos suficientes que les deben permitir tomar decisiones éticas delante los dilemas complejos que se van a encontrar en su práctica profesional (DEL RÍO; MIRÓ , 2002; DEL RÍO; BORDA; TORRES, 2003).

En relación con lo anteriormente anotado la dimensión ética de la práctica profesional debería convertirse en un objetivo formativo prioritario del practicum en Psicología (VIÑAS, et al., 2007).

Rev. Diálogo Educ., Curitiba, v. 9, n. 26, p. 91-101, jan./ abr. 2009 
A continuación presentamos un curso, en forma de taller, que pretende, precisamente, abordar algunas situaciones prácticas donde entran en juego principios éticos que los estudiantes se pueden encontrar una vez ejerzan su profesión (Esteban, 2008). El taller tiene tres objetivos:

a) conocimiento de las bases epistemológicas de la ética;

b) identificación y análisis de problemáticas éticas asociadas a la práctica profesional;

c) pazonamiento ético y presa de decisiones a partir del análisis de casos y la simulación del funcionamiento de un comité de ética aplicada.

El taller o programa formativo consta de 11 sesiones de hora y media (un total de 16 horas y media) repartidas del siguiente modo:

1) sesión introductoria donde se explica el objetivo del taller, así como se revisa y analiza el código deontológico a partir de la vulneración de distintos artículos recogidos por el Colegio O ficial de Psicólogos de España;

2) dos sesiones dedicadas a las bases epistemológicas de la ética, así como a introducir la ética aplicada y el concepto de "dilemas éticos" (dos o más principios o juicios normativos puestos en contradicción). En esta sesión se encarga la redacción, por parte de los estudiantes, de una situación práctica en la cual se ilustre un "dilema ético";

3) dos sesiones dedicadas a los conceptos e instrumentos jurídicos que enmarcan la praxis profesional del psicólogo;

4) una sesión donde se ilustra los instrumentos, metodología y protocolos para la resolución de problemas éticos mediante la simulación de un Comité de Ética Asistencial y un Comité de Ética de Investigación Clínica. Los estudiantes entregan los ejercicios (los dilemas éticos que han redactado);

5) tres sesiones donde distintos profesionales de la psicología (uno que trabaja en el contexto socioeducativo; otro en el de las organizaciones y psicología del trabajo y otro en el campo de intervención clínica) hablan y exponen dilemas éticos;

6) finalmente, dos últimas sesiones que analizan, valoran y resuelven los casos presentados por los alumnos;

Rev. D iálogo Educ., Curitiba, v. 9, n. 26, p. 91-101, jan./ abr. 2009 
Siguiendo otros programas de intervención o formación (PÉREZ; CABEZAS, 2007) se trata de asumir los tres objetivos anteriormente expuestos a partir del entrenamiento en solución de problemas prácticos donde los estudiantes se exponen, de una manera vicaria (BAND URA; WALTERS, 1987), a distintos ejemplos y modelos que les permiten conocer el código deontológico; identificar dilemas éticos y evaluar soluciones optimas.

Con el objetivo de comprobar la eficacia, o no, del mencionado programa o taller formativo se diseñó, inspirándonos en otros trabajos (BORDA et al., 2002; DEL RÍO et al., 2003; HERMO SILLA et al., 2007; LOZANO, 2002), un cuestionario en el que se plantearon 5 situaciones basadas en distintos artículos que aparecen en el Código D eontológico. Dicho cuestionario se administró a los estudiantes de quinto de psicología antes y después del entrenamiento con el fin de determinar cambios en las respuestas obtenidas. La hipótesis de investigación es la siguiente: se esperan encontrar diferencias estadísticamente significativas antes y después de participar en el taller de modo que prevalecerá una mayor cantidad de juicios éticamente correctos en la aplicación del cuestionario después de asistir a las distintas sesiones del taller o programa de entrenamiento.

\section{MÉTODO}

\section{Participantes}

En el estudio participaron 51 estudiantes de 5o de la Licenciatura de Psicología, que estaban cursando el practicum. El curso o taller es de asistencia obligatoria de modo que participaron todos los matriculados y matriculadas al último curso de psicología que realizaban el practicum.

\section{Instrumento}

Se diseñó un cuestionario en el que se plantearon 5 situaciones que implicaban juicios éticos. La situación 5 se extrajo del trabajo de D el Río, et al. (2003, p. 61). En función del código deontológico (artículos que aparecen en el apéndice) se establecieron 5 opciones éticamente correctas (respetan el código deontológico) y 5 opciones incorrectas. Por ejemplo, la situación 1 dice: Un naciente entra en terapia y a lo largo de las suœesivas entrevistas nos cuenta que está padeciendo abusos sex uales por parte de su pareja. ¿Q ué hacemos?. Se dan dos opciones de respuesta: a) respetamos el "secreto profesional" y de ningún modo decimos nada a nadie; b) informamos, como mínimo, a los organismos colegiales. Según el Código D eontológico la respuesta correcta es la b ya que, siguiendo el artículo 8:

Rev. Diálogo Educ., Curitiba, v. 9, n. 26, p. 91-101, jan./ abr. 2009 
Todo/a Psicólogo/a deber informar, al menos a los organismos colegiales, acerca de violaciones delos derechos humanos, malos tratos o condiciones de reclusión crueles, inhumanas o degradantes de que sea víctima cualquier persona y de los que tuviere conocimiento en el ejercicio de su profesión. (COP, 2004, p. 112).

Cabe señalar que la instrucción que acompañaba el cuestionario era la siguiente: A continuación se presentan 5 situaciones que debes valorar, des del punto de vista ético, según te parezcan, o no, orrectas. Para eso señala on una "E" las situaciones éticamente correctas y con una "I" aquellas que te parezcan incorrectas. E n cada situación puede haber 1 situación ética (E ) y una no ética (I); 2 éticas (E y E ) 02 incorrectas (I y I).

\section{Procedimiento}

Antes de la realización de las sesiones que confeccionan el programa o taller sobre ética profesional se aplicó el cuestionario a 51 estudiantes (momento temporal 1 o pretest). Una vez realizada la intervención (las 16 horas y media de curso) se volvió a aplicar el mismo cuestionario (momento temporal 2 o postest). Cabe aclarar que en ningún momento del transcurso del taller se corrigió el cuestionario de modo que los estudiantes no sabían, al finalizar el pretest, si las respuestas eran correctas o incorrectas. Así mismo los profesionales que ejercían de docentes en las sucesivas sesiones desconocían el cuestionario administrado y las situaciones que en el mismo se explicitaban.

\section{RESULTADOS}

Se realiza una prueba T para muestras relacionadas con el estadístico $\mathrm{t}$ de Student asociada para comprobar la significación de una diferencia de medias. El diseño seguido ha sido apareado, es decir, los mismos individuos son observados antes y después de una determinada intervención, en nuestro caso antes y después de las distintas sesiones que componen el taller de ética aplicada.

En la Tabla 1 se observa la media del total de respuestas correctas en el pretest $(7,78)$ y en el postest $(9,24)$. El rango va de 0 a 10 de modo que puntuaciones más próximas a 10 indican un número mayor de respuestas correctas. Se observan diferencias estadísticamente significativas entre ambas medias $(t=$ 8,160; $p<0.005)$, siendo la del postest más elevada.

Rev. Diálogo Educ., Curitiba, v. 9, n. 26, p. 91-101, jan./ abr. 2009 
ESTEBAN GUITART, $\mathrm{M}$.

TABLA 1 - Comparación de puntuaciones

Correctas en el pre y el postest

\begin{tabular}{lcccc}
\hline & Media & D.S. & t & Sig. (rango 0 - 10) \\
\hline $\begin{array}{l}\text { Total } \\
\text { pretest }\end{array}$ & 7,78 & 1,045 & & \\
Total & 9,24 &, 815 & &, 000 \\
Postest & & & & \\
\hline
\end{tabular}

Observando las respuestas a y b de cada situación planteado (un total de cinco situaciones) observamos que las medias son siempre mayores en el postest comparado con las medias del pretest (ver Figura 1).

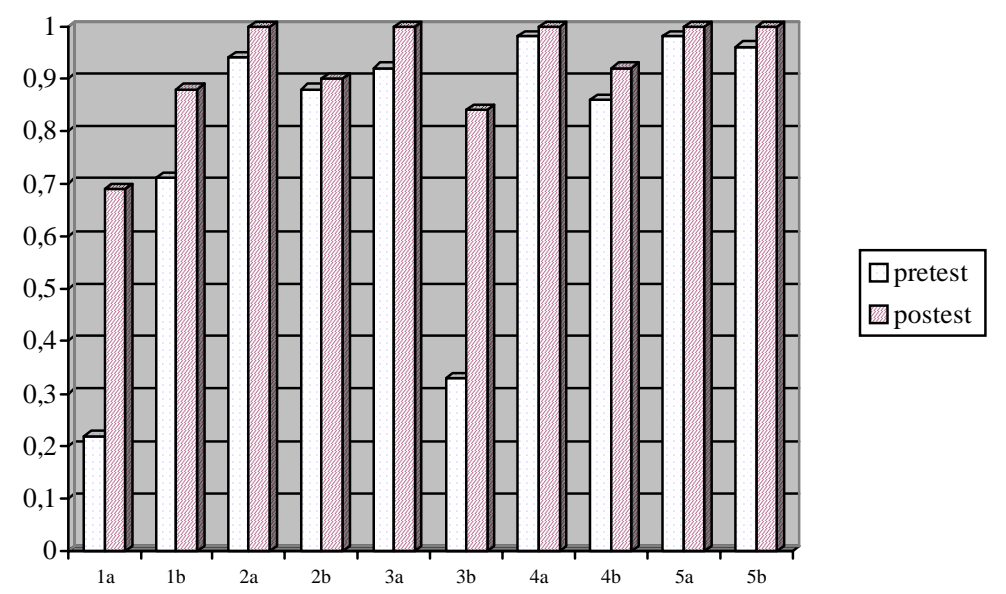

FIG URA 1 - Media de puntuaciones (rango = 0-1) en las distintas preguntas antes y después de la intervencion

Ello es especialmente destacado en las situaciones 1 y 3 donde se observan diferencias estadísticamente significativas en la media de puntuaciones correctas en las preguntas 1a; 1b; 3a; y 3b (ver Tabla 2).

Rev. Diálogo Educ., Curitiba, v. 9, n. 26, p. 91-101, jan./ abr. 2009 
TABLA 2 - Comparación pre - postest en las 5 preguntas

\begin{tabular}{|c|c|c|c|c|c|}
\hline \multirow[t]{2}{*}{ Situación } & \multirow[t]{2}{*}{ Pregunta } & \multicolumn{2}{|c|}{$\begin{array}{c}\text { Media } \\
\text { (rango 0-1) }\end{array}$} & \multirow[t]{2}{*}{$\mathbf{t}$} & \multirow[t]{2}{*}{ Sig. } \\
\hline & & Pretest & Postest & & \\
\hline \multirow[t]{2}{*}{1} & 1a & 0,22 & 0,69 & $-5,814$ &, $000^{*}$ \\
\hline & $1 \mathrm{~b}$ & 0,71 & 0,88 & $-2,433$ & ,019* \\
\hline \multirow[t]{2}{*}{2} & $2 a$ & 0,94 & 1 & $-1,768$ & ,083 \\
\hline & $2 b$ & 0,88 & 0,9 &,- 375 & ,709 \\
\hline \multirow[t]{2}{*}{3} & 3а & 0,92 & 1 & $-2,063$ &, $044^{*}$ \\
\hline & $3 b$ & 0,33 & 0,84 & $-6,291$ &, $000^{*}$ \\
\hline \multirow[t]{2}{*}{4} & $4 a$ & 0,98 & 1 & -1 & ,322 \\
\hline & $4 b$ & 0,86 & 0,92 &,- 903 & ,371 \\
\hline \multirow[t]{2}{*}{5} & $5 a$ & 0,98 & 1 & -1 & 322 \\
\hline & $5 b$ & 0,96 & 1 & $-1,429$ & 159 \\
\hline
\end{tabular}

En el pretest de la pregunta 1a se observa un $22 \%$ de respuestas correctas frente un $69 \%$ del postest. A pesar de la mejoría es la pregunta que presenta un número inferior de respuestas correctas. Es decir, algunos estudiantes, delante la situación 1, piensan que lo más correcto es mantener el "secreto profesional". En la pregunta $1 \mathrm{~b}$ también aumenta el porcentaje de respuestas correctas a pesar que la diferencia no es tan destacado (de un $71 \%$ se pasa a un $88 \%$ ).

En las preguntas referidas a la situación 2 también se muestran mejoras, en este caso no significativas, entre las medias del pretest y las del postest. En este caso se pasa de un $94 \%$ a un $100 \%$ en la pregunta 2a y de un $88 \%$ a un $90 \%$ en la pregunta $2 \mathrm{~b}$.

Por lo que respecta a la situación 3 las diferencias entre las medias, antes y después de la intervención, son significativas tanto en la pregunta 3a (se pasa de un $92 \%$ a un 100\%) como, sobre todo, en la pregunta 3b donde se pasa de un 33\% de respuestas correctas a un $84 \%$.

Finalmente, tanto en las preguntas referidas a las situación 4 como aquellas vinculadas con la situación 5 el aumento de medias del postest no es significativo. Todas ellas, menos la pregunta $4 \mathrm{~b}$ que pasa de un $86 \%$ a un $92 \%$ de acierto, llegan al 100\% en las puntuaciones del postest, es decir, todos los estudiantes responden correctamente.

Rev. Diálogo Educ., Curitiba, v. 9, n. 26, p. 91-101, jan./ abr. 2009 


\section{DISCUSIÓN}

Al iniciar el trabajo nos proponíamos presentar y evaluar un programa formativo en ética profesional en el marco del practicum delos estudios de psicología. Esperábamos encontrar diferencias estadísticamente significativas entre los juicios o respuestas éticamente correctas en el cuestionario aplicado antes y después de la realización de las 11 sesiones que conforman el seminario o taller. Los resultados confirman dicha hipótesis. Las puntuaciones son mejores en el postest en comparación al pretest. Ello es especialmente significativo en las situaciones 1 y 3 .

En la situación 1 se presenta un dilema ético que contrapone el "secreto profesional" con la integridad de la persona que entra en terapia (D EL RÍO, 2007). Por una parte el artículo 40 del Código Deontológico establece el deber y el derecho de mantener el "secreto profesional" en todas aquellas informaciones recogidas en el ejercicio profesional. Por otro lado, el artículo 8, como ya ha sido apuntado anteriormente, obliga al psicóloga/ a a informar, al menos a los organismos colegiales, de aquellos casos donde se están violando los derechos humanos (como en la situación de malos tratos que se describe en la historia 1). Probablemente la mejor intervención seaintentar convencer a la persona que realice las medidas convenientes con el objetivo de superar la situación (por ejemplo, informar, ella misma, a la policía). D e esta manera se mantiene el "secreto profesional". De todos modos, Código Deontológico en mano, la pregunta 1a es incorrecta mientras que la pregunta $1 \mathrm{~b}$ es correcta. Al finalizar las sesiones un 69\% (pregunta 1a) y un 88\% (para la pregunta 1b) de los estudiantes responde de acuerdo a las indicaciones del Código D eontológico. La mejora es destacable ya que se partía de los porcentajes de acierto más bajos teniendo presente todas las medias del pretest $(0,22$ y 0,71, respectivamente). La situación 1 representa un ejemplo de lo que supone encontrarse con un dilema ético, es decir, tomar una decisión frente a dos principios normativos contradictorios o que nos inclinan a adoptar dos actitudes opuestas (por un lado no decir nada, manteniendo el secreto profesional, por el otro decirlo, manteniendo la integridad de la persona que entra en consulta) (DEL RÍO, 2007; HERMO SILLA et al., 2006).

La situación 2 plantea una posible presentación delanteun determinado barrio o colectivo en el que realizamos una determinada intervención. Según el artículo 25 al hacerse cargo de una intervención sobre personas, grupos, instituciones o comunidades el o la psicólogo/ a debe ofrecer información adecuada sobre las características de la relación que se va a establecer, los problemas que se abordaran, los métodos utilizados así como los objetivos que se proponen. Un 94\% de los estudiantes parecen tener clara esta indicación en el pretest. Puntuación que llega al $100 \%$ en el postest. En la pregunta $2 \mathrm{~b}$ la diferencia también es ligera (de un 88\% de respuestas correctas a un 90\%). Permanece, no obstante, un $10 \%$ que piensa que lo

Rev. Diálogo Educ., Curitiba, v. 9, n. 26, p. 91-101, jan./ abr. 2009 
éticamente correcto es esconder los objetivos y la profesión para evitar generar recelos en las personas afectadas por nuestra intervención. Ello conllevaría una vulneración del artículo 25 del Código D eontológico del psicólogo/ a.

En la situación 3 se expone un caso donde nuestro posible paciente asiste a terapia con otro profesional. Mientras que no podemos interferir en las intervenciones iniciadas por el otro o la otra psicólogo/ a (según el artículo 30), si que podemos negarnos a aceptar la simultaneidad de ambas terapias (artículo 27). Mientras que la mayor parte de estudiantes responden correctamente a la pregunta 3a, evitando interferir en la terapia de otros profesionales, solamente un 33\% de los estudiantes sabía, antes de realizar el curso de ética aplicada, que se podía negar a aceptar la simultaneidad de ambas terapias. Porcentaje que sube al 84\% después de la realización del seminario o taller formativo en ética aplicada. Ello nos inclina a pensar que al finalizar la carrera algunos estudiantes desconocen aspectos del Código D eontológico como, por ejemplo en el caso analizado, la posibilidad de negarse a realizar simultaneidad terapéutica (DEL RÍO; MIRÓ, 2002).

Lasituación 4y, penúltima, plantea el tema de lacompetenciaprofesional. Según dice el artículo 17 la autoridad profesional del psicólogo/ a se fundamenta en su capacitación y calificación para las tareas que desempeña. Entre ellas el conocimiento y preparación suficiente que permita un buen uso de los instrumentos, técnicas y procedimientos que adopte en su trabajo. La gran mayoría de estudiantes de nuestro estudio dicen que la respuesta correcta sería realizar un curso de capacitación homologada para utilizar correctamente el instrumento. A pesar de ello un 14\% delos estudiantes pensaba, antes de realizar el curso, que era éticamente correcto utilizar un cuestionario de personalidad a partir de una simple lectura en internet.

Finalmente la última situación planteada se enmarca dentro de la problemática alrededor de las "relaciones duales" (DEL RÍO et al., 2003), es decir, cuando el terapeuta se encuentra manteniendo, además de la terapéutica, otra relación diferente con un paciente que puede ser social (sexual o no sexual), profesional, financiera, etc. Un alto porcentaje de estudiantes, que en el postest llega al $100 \%$, responden correctamente en las preguntas $5 a$ y $5 \mathrm{~b}$. Según los artículos 11 y 29 del Código Deontológico el psicólogo o la psicóloga no aprovechará, para lucro o beneficio propio o de terceros, la situación de poder o superioridad que pueda derivarse de su profesión, así como no se prestará a situaciones confusas donde su papel o función sean ambiguas. Delante la misma situación planteada en otra muestra de estudiantes D el Río et al. (2003) encuentran que un 12,5\% de los estudiantes accederían a mantener relaciones sexuales dentro de la consulta durante el horario de la terapia indicando, en consecuencia, que un considerable porcentaje de alumnos optaría por una respuesta que plantea problemas éticos. En nuestro caso, como se ha indicado ya, en el postest ningún estudiante dice acceder a mantener relaciones sexuales dentro de la consulta.

Rev. Diálogo Educ., Curitiba, v. 9, n. 26, p. 91-101, jan./ abr. 2009 
A lo largo del análisis de las distintas situaciones planteadas a los estudiantes de $5^{\underline{0}}$ curso de psicología de nuestra muestra se pone de manifiesto que existen importantes mejoras en la identificación y resolución de los problemas éticos derivados del ejercicio profesional después de realizar el curso o seminario sobre ética aplicada a los ámbitos profesionales del psicólogo/ a. Ello da apoyo empírico a la eficacia del curso que, al igual que otros modelos educativos o de intervención (PÉREZ; CABEZAS, 2007), se basa en el planteamiento y resolución de problemas y casos prácticos (para ver una sesión ilustrativa se puede consultar ESTEBAN, 2008). Con la ayuda de profesionales de la psicología implicados en sus distintos ámbitos de aplicación (socioeducativa; sociosanitaria o clínica y psicología del trabajo y las organizaciones) los estudiantes se familiarizan con distintos casos reales y aprenden a identificary analizar los problemas éticos asociadas a la práctica profesional.

No obstante el trabajo presenta distintas limitaciones. Una de ellas y, pensamos que es la principal, el escaso número de estudiantes que componen la muestra y que nos obliga a ser prudentes en la generalización de los resultados y conclusiones. O tra es el modo de evaluación de la eficacia del programa. A pesar de que se trata de un diseño transversal pretest - postest, lo que asegura observar los cambios consecuencia de una determinada intervención, las situaciones examinadas son cinco de modo que no abarcan otras posibles realidades y problemáticas que se reflejan en el Código D eontológico. Para superar ambas limitaciones estudios posteriores deberían ampliar la muestra objeto de estudio e incluir más situaciones en los procedimientos de evaluación.

El Colegio O ficial de Psicólogos (2004) establece que el conocimiento y cumplimiento del código deontológico es un requisito imprescindible y necesario para el ejercicio profesional del psicólogo o la psicóloga en los distintos ámbitos de aplicación. Para ello las Universidades deben ofrecer una formación adecuada a sus estudiantes de modo que los futuros profesionales de la psicología conozcan el código deontológico que rige las coordenadas bajo las cuales debe moverse su actuación profesional. Esperamos que el modelo educativo esbozado pueda sugerir pistas e ideas acerca de modos de trabajar cuestiones éticas en el contexto del practicum de los estudios de psicología.

\title{
REFERENCIAS
}

\author{
BANDURA, A.; WALTERS, R. H. Aprendizaje social y desarrollo \\ de la personalidad. Madrid: Alianza, 1987.
}

BO RDA, M; et al. MARTÍN, A. ¿Conocen los estudiantes de Psicología los principios éticos básicos para actuar como terapeutas? Revista de Enseñanza Universitaria, v. 19, p. 15-43, 2002.

Rev. Diálogo Educ., Curitiba, v. 9, n. 26, p. 91-101, jan./ abr. 2009 
COLEG IO OFICIAL DE PSICÓLOGOS. Ética y deontología para psicólogos. Madrid: Colegio O ficial de Psicólogos de España, 2004.

DEL RÍO, C.; MIRÓ, J. La docencia de la ética profesional para psicólogos. Revista de Psicología. Universitas Tarraconensis, v. 24, p. 9-19, 2002.

DEL RÍO, C.; BO RDA, M.; TO RRE S, I. Valoración de los estudiantes de Psicología sobre la ética de algunas prácticas de los terapeutas. Psicología Conductual, v. 11, n. 2, p. 261-281, 2003.

DEL RÍO, C; et al.Ética de las relaciones duales en psicoterapia. Psicothema, v. 15, p. 58-64, 2003.

DEL RÍO, C. Dilemas éticos relacionados con la confidencialidad. Información Psicológica, v. 90, p. 12-27, 2007.

ESTEBAN, M. Uso de problemáticas éticas para la enseñanza del Código D eontológico de la psicología. Una sesión ilustrativa. RE P.TE. Revista de E nseñanza de la Psicología: Teoría y Experiencia, v. 4, p. 14-20, 2008.

HERMO SILLA, A. M; et al. Dilemas éticos en el ejercicio de la psicología: resultados de una investigación. Fundamentos en humanidades, v. 7, n. 1 y 2, p. 91-106, 2006.

LOZANO, Luis. Dilemas éticos en el trabajo con menores. Revista de Psicología. Universitas Tarraconensis, v. 24, n. 1 y 2, p. 41-57, 2002.

PÉREZ, L.; CABEZAS, D. Programa de entrenamiento en solución de problemas prácticos aplicado a personas con discapacidad intelectual. Psicothema, v. 19, n. 4, p. 578-584, 2007.

VIÑAS, F; et al. La dimensión ètica de la pràctica professional. In: VILLAR, E. (Ed.). Pràcticum de psicologia: fonaments, reflexions i propostes. Girona: D ocumenta Universitaria, 2007. p. 209-240.

Recebido: 30/ 08/ 2008

Received: 08/ 30/ 2008

Aprovado: 10/ 11/ 2008

A pproved: $11 / 10 / 2008$

Rev. Diálogo Educ., Curitiba, v. 9, n. 26, p. 91-101, jan./ abr. 2009 